\title{
Intermittent maser flare around the high-mass young stellar object G353.273+0.641
}

\author{
Kazuhito Motogi ${ }^{1}$, Kazuo Sorai ${ }^{1,2}$, Kenta Fujisawa ${ }^{3,4}$, \\ Koichiro Sugiyama ${ }^{3}$ and Mareki Honma ${ }^{5,6}$ \\ ${ }^{1}$ Department of Cosmosciences, Graduate School of Science, Hokkaido University, \\ N10 W8, Sapporo 060-0810, Japan \\ email: motogi@astro1.sci.hokudai.ac.jp \\ ${ }^{2}$ Department of Physics, Faculty of Science, Hokkaido University, \\ N10 W8, Sapporo 060-0810, Japan \\ ${ }^{3}$ Department of Physics, Faculty of Science, Yamaguchi University, \\ Yoshida 1677-1, Yamaguchi-city, Yamaguchi 753-8512 2, Japan \\ ${ }^{4}$ The Research Institute of Time Studies, Yamaguchi University, \\ Yoshida 1677-1, Yamaguchi-city, Yamaguchi 753-8511, Japan \\ ${ }^{5}$ Department of Astronomical Science, The Graduate University for Advanced Studies, \\ 2-21-1 Osawa, Mitaka, Tokyo 181-8588, Japan \\ ${ }^{6}$ Mizusawa VLBI Observatory, National Astronomical Observatory of Japan, \\ 2-12 Hoshi-ga-oka, Mizusawa-ku, Oshu, Iwate 023-0861, Japan
}

\begin{abstract}
The water maser site associated with G353.273+0.641 is classified as a dominant blueshifted $\mathrm{H}_{2} \mathrm{O}$ maser, which shows an extremely wide velocity range $\left( \pm 100 \mathrm{~km} \mathrm{~s}^{-1}\right)$ with almost all flux concentrated in the highly blueshifted emission. The previous study has proposed that this peculiar $\mathrm{H}_{2} \mathrm{O}$ maser site is excited by a pole-on jet from high mass protostellar object. We report on the monitoring of $22-\mathrm{GHz} \mathrm{H}_{2} \mathrm{O}$ maser emission from $\mathrm{G} 353.273+0.641$ with the VLBI Exploration of Radio Astrometry (VERA) and the Tomakamai 11-m radio telescope. Our VLBI imaging has shown that all maser features are distributed within a very small area of $200 \times 200 \mathrm{au}^{2}$, in spite of the wide velocity range $\left(>100 \mathrm{~km} \mathrm{~s}^{-1}\right)$. The light curve obtained by weekly single-dish monitoring shows notably intermittent variation. We have detected three maser flares during three years. Frequent VLBI monitoring has revealed that these flare activities have been accompanied by a significant change of the maser alignments. We have also detected synchronized linear acceleration $\left(-5 \mathrm{~km} \mathrm{~s}^{-1} \mathrm{yr}^{-1}\right)$ of two isolated velocity components, suggesting a lower-limit momentum rate of $10^{-3} \mathrm{M}_{\odot} \mathrm{km} \mathrm{s}^{-1} \mathrm{yr}^{-1}$ for the maser acceleration. All our results support the previously proposed pole-on jet scenario, and finally, a radio jet itself has been detected in our follow-up ATCA observation. If highly intermittent maser flares directly reflect episodic jet-launchings, G353.273+0.641 and similar dominant blueshifted water maser sources can be suitable targets for a time-resolved study of high mass protostellar jet.
\end{abstract}

Keywords. masers, stars: early-type, stars: formation, ISM: jets and outflows

\section{Introduction}

G353.273+0.641 (hereafter G353) is a strong $22 \mathrm{GHz} \mathrm{H}_{2} \mathrm{O}$ maser site in the southern high mass star-forming region NGC6357 (Skellis et al. 1984). The source distance is $1.7 \mathrm{kpc}$ from the sun (Neckel 1978). Multi-epoch ATCA observation has been reported in Caswell \& Phillips (2008) (hereafter CP08). Class II $\mathrm{CH}_{3} \mathrm{OH}$ maser emission $\left(J_{k}=\right.$ $5_{1}-6_{0} \mathrm{~A}^{+}$) at $6.668519 \mathrm{GHz}$ is also associated with this source (CP08), and hence, the host young stellar object (YSO) is identified as a high mass YSO (e.g., Minier et al. 2003). CP08 has also suggested that G353 is still in the pre-ultra compact (UC) H II 

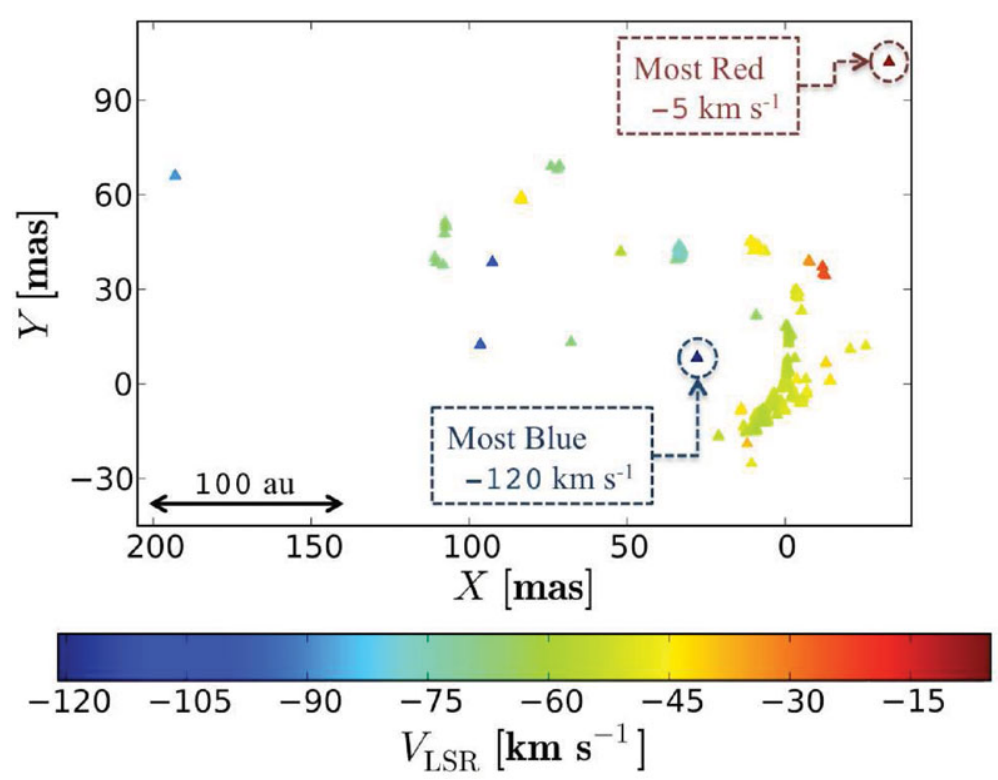

Figure 1. Spatial distribution of detected maser spots. Here, each triangle indicates detected maser spot and its color represents a line of sight velocity. The coordinate origin is $\alpha_{2000}=17^{\mathrm{h}} 26^{\mathrm{m}} 01^{\mathrm{s}} .5883, \delta_{2000}=-34^{\circ} 15^{\prime} 14^{\prime \prime} .905$.

region phase, i.e., high mass protostellar phase, based on the absence of any detectable OH masers (e.g., Caswell 1997; Breen et al. 2010).

G353 has been classified as a dominant blue-shifted $\mathrm{H}_{2} \mathrm{O}$ maser in CP08. That is, almost all flux is concentrated in the blue-shifted emission, despite the very broad velocity range of $\pm 100 \mathrm{~km} \mathrm{~s}^{-1}$ with respect to the systemic velocity of $-5 \mathrm{~km} \mathrm{~s}^{-1}$. They argued that this type of masers can be caused by well-collimated jet aligned close to the line of sight. There are a few maser sources which show similar blue-shift dominance (e.g., Caswell 2004; CP08; Caswell \& Breen 2010). Some of them suggest acceleration of outflowing materials in the jet. The statistical analysis in Caswell \& Breen (2010) indicates that such a blue-shift dominance is a characteristic of $\mathrm{H}_{2} \mathrm{O}$ masers at the earliest evolutionary stage of star-formation.

The exact relation between jet activity and variability of the maser is still unclear, but, once they appear, $\mathrm{H}_{2} \mathrm{O}$ masers are an excellent tool to survey dynamic jet activities in small scale, since its bright emission allows us easy and frequent monitoring even with a small size radio telescope.

\section{Long-term monitoring of the $\mathrm{H}_{2} \mathrm{O}$ maser}

Our VLBI and single dish monitoring of G353 using VERA (VLBI Exploration of Radio Astrometry) and the Hokkaido University Tomakomai 11-m radio telescope, which has been started in 2008 November and is still ongoing, has shown intermittent flare activities (see Motogi et al. 2011 in details). Figure 1 presents overall distribution of maser spots. We have detected only the blueshifted side of the entire maser emission reported in CP08. The most blueshifted and redshifted components are separated by only 100 mas (200 au) along the SE-NW direction.

Figure 2 shows the light-curve of the main velocity components $\left(-53 \pm 7 \mathrm{~km} \mathrm{~s}^{-1}\right)$. The observed variation is notably intermittent and there are three significant maser flares. Motogi et al. (2011) has revealed that these flares are accompanied by spatial varia- 


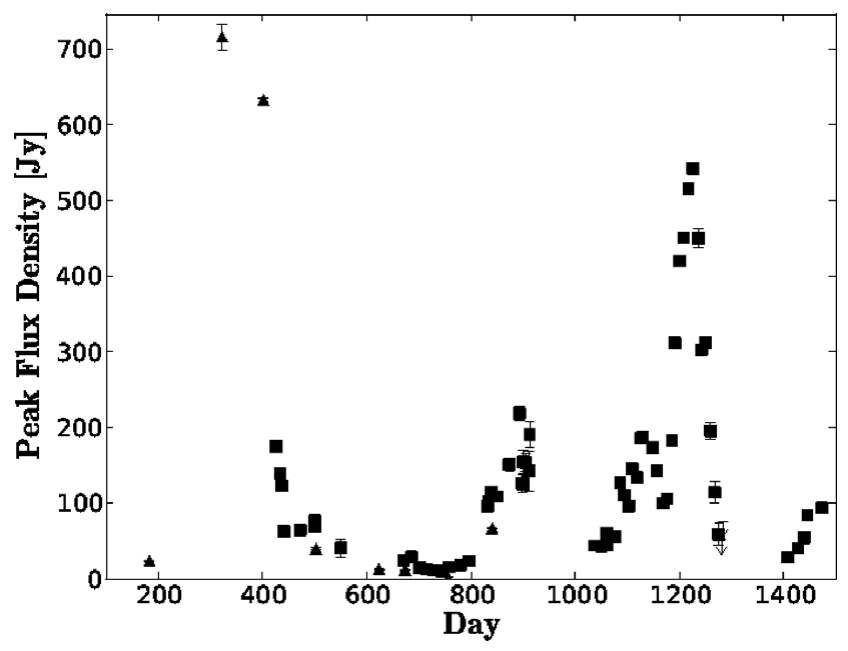

Figure 2. The light curve of the maser components at the line of sight velocity of $-53 \pm 7 \mathrm{~km}$ $\mathrm{s}^{-1}$. The $\mathrm{x}$-axis show relative days from the first day of 2008 . The squares and triangles show single dish and VLBI data points, respectively. Follow-up single-dish data, which show the 3rd flare, are added to the dataset presented in Motogi et al. (2011)

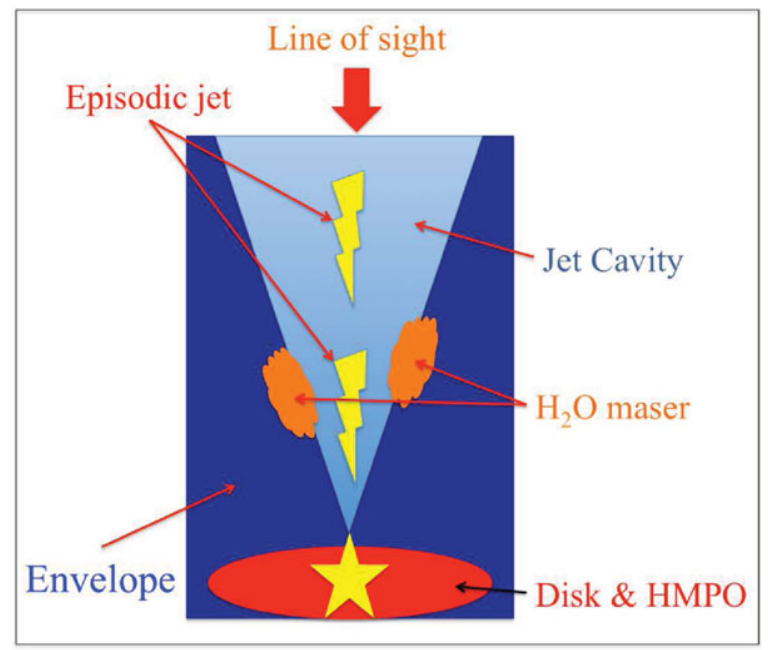

Figure 3. Schematic view of the "pole-on jet" model in G353.

tions, which can be explained by episodic shock propagation along the SE-NW direction. The characteristic velocity, which is estimated from the spatial scale of maser distribution ( $\sim 100 \mathrm{au})$ divided by the time interval of three times flares $(\sim 1 \mathrm{yr})$, is $\sim 500 \mathrm{~km} \mathrm{~s}^{-1}$. This is rather larger than the typical three-dimensional velocity of this maser site $\left(\sim 100 \mathrm{~km} \mathrm{~s}^{-1}\right)$, and more like that of a radio jet seen in several HMPOs (Curiel et al. 2006; Martí, Rodrígues \& Reipurth 1998). Motogi et al. (2011) has also measured the linear acceleration $\left(5 \mathrm{~km} \mathrm{~s}^{-1} \mathrm{yr}^{-1}\right)$ of two distinct maser clusters (see Motogi et al. 2011 in detail). The lower-limit momentum rate required for the acceleration is $\sim 1.1 \times 10^{-3} \mathrm{M}_{\odot} \mathrm{km}$ $\mathrm{s}^{-1} \mathrm{yr}^{-1}$. This value is consistent with that of the outflows driven by high mass YSOs (Arce et al. 2007). 


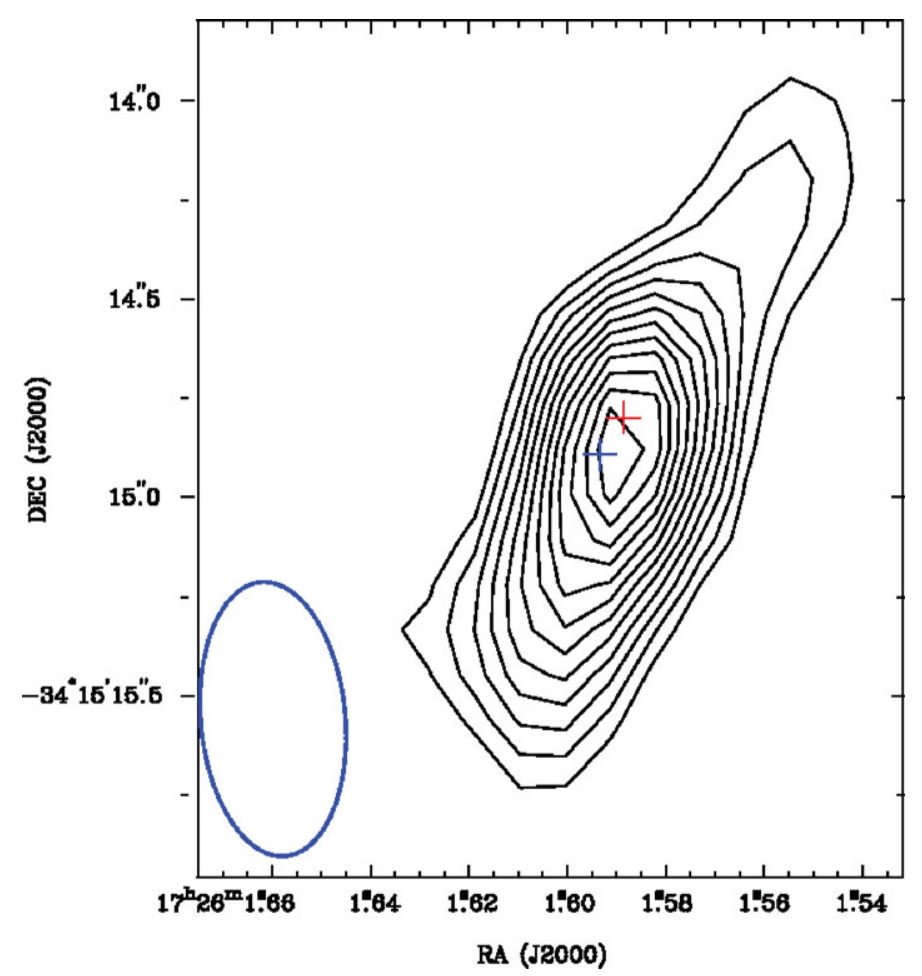

Figure 4. ATCA contour image of $22 \mathrm{GHz}$ radio continuum emission from G353. Contour levels are 105 to $545 \mu \mathrm{Jy}_{\text {beam }}{ }^{-1}$ in steps of $40 \mu \mathrm{Jy}_{\text {beam }}{ }^{-1}$. The lowest level corresponds to 3 $\sigma$ noise. The synthesized beam $\left(0^{\prime \prime} .69 \times 0^{\prime \prime} .36\right)$ is shown in the lower left corner. The most red and blue maser components are shown by red and blue cross, respectively. Relative positional error between masers and continuum is less than $0^{\prime \prime} .1$.

\section{Follow-up jet survey}

Since all maser properties can be consistently explained by the "pole-on jet" model (see figure 3), we have finally performed direct survey of a radio jet itself using the Australia Telescope Compact Array (ATCA). Our ATCA radio continuum observation was made at January 132012 in the $6 \mathrm{~A}$ configuration. We observed two $2 \mathrm{GHz}$ bands centered on 18 and $22 \mathrm{GHz}$ simultaneously with the Compact Array Broadband Backend (CABB; Wilson et al. 2011). The observed hour-angle range is 10 hour and total on-source time is 6 hour because of fast switching (the cycle time of $3 \mathrm{~min}$ ). The $1-\sigma$ noise level is 19 and $35 \mu \mathrm{Jy}$ beam $^{-1}$ for 18 and $22 \mathrm{GHz}$, respectively.

As a result, we have successfully detected a weak and optically thick thermal radio jet (Motogi et al. 2012, in prep). Figure 4 shows ATCA contour image of $22 \mathrm{GHz}$ continuum emission. The spectral index estimated from the total flux of 18 and $22 \mathrm{GHz}$ continuum is about +1.5 (see table 1 ) and is consistent with that of a typical radio jet from an HMPO (e.g., Hofner et al. 2007). The jet is clearly elongated along the NW - SE direction, indicating that the jet has a finite inclination angle. This direction is consistent with the direction of the velocity gradient and shock propagation shown by our maser monitoring. This fact strongly suggests physical association between the maser and jet activities. 
Table 1. Properties of the detected radio jet

\begin{tabular}{cccc}
\hline $\begin{array}{c}\text { Frequency } \\
(\mathrm{GHz})\end{array}$ & $\begin{array}{c}\text { Peak Flux } \\
\left(\mathrm{mJy} \mathrm{beam}^{-1}\right)\end{array}$ & $\begin{array}{c}\text { Total Flux } \\
(\mathrm{mJy})\end{array}$ & Spectral Index \\
\hline 18 & $0.54 \pm 0.02$ & $0.76 \pm 0.07$ & +1.5 \\
22 & $0.59 \pm 0.04$ & $1.02 \pm 0.11$ & \\
\hline
\end{tabular}

\section{Future Works}

Our studies have validated the "pole-on jet" model for the dominant blueshifted water masers, and we are now planning further follow-up studies in order to reveal exact relation between $\mathrm{H}_{2} \mathrm{O}$ maser and jet activities. Simultaneous proper motion measurements of the masers are, especially, a direct way to examine their relation. Careful comparison between the maser light-curve and propagation of the jet is also valuable, because such a direct correlation between a maser and thermal emission in the time-domain have not yet been reported in a star-forming region. It will be a good example of what expected in extremely high-resolution studies of a high mass protostellar system in the ALMA era.

Furthermore, if highly intermittent variation in the maser-light curve actually traces episodic jet-launchings, then a G353-type $\mathrm{H}_{2} \mathrm{O}$ maser source can be a suitable target for a time-resolved study of high mass protostellar jet. Single-dish based, dense maser monitoring can give us not only a unique statistical dataset about jet-variation, but also a chance to survey any time variation in the innermost region of an accretion disk along jet-launching activities.

\section{Acknowledgements}

This work was financially supported by the Grant-in-Aid for the Japan Society for the Promotion of Scinence Fellows (KM).

\section{References}

Arce, H. G., et al. 2007, in Reipurth, B., Jewitt, D., \& Keil, K., eds, Protostars and Planets V. Univ. of Arizona Press, Tucson, p. 245

Breen, S. L., Caswell J. L., Ellingsen, S. P., \& Phillips, C. J. 2010, MNRAS, 406, 1487

Caswell J. L. 1997, MNRAS, 209, 203

Caswell J. L. 2004, MNRAS, 351, 279

Caswell J. L. \& Phillips C. J. 2008, MNRAS, 386, 1521 (CP08)

Caswell J. L. \& Breen S. L. 2010, MNRAS, 407, 2599

Curiel S., et al. 2006, ApJ, 638, 878

Hofner, P., Cesaroni, R., Olmi, L., Rodrígues, L. F., Martí, J. \& Araya, E. 2007, A\&A, 465, 197

Martí, J., Rodrígues, L. F., \& Reipurth, B. 1998, ApJ, 502, 337

Minier, V., Ellingsen, S. P., Norris, R. P. \& Booth, R. S. 2003, A\&A A, 403, 1095

Motogi, K., et al. 2011, MNRAS, 417, 238

Neckel, T. 1978, A\&A, 69, 51

Sakellis, S., Taylor, M. I., Taylor, K. N. R., Vaile, K. A., \& Han, T. D. 1984, PASP, 96, 543

Wilson, W. E., et al. 2011, MNRAS, 416, 832 